\title{
Influence of the characteristics of a light source and target on the Monge-Ampére equation method in freeform optics design
}

\author{
Rengmao Wu, Pablo Benítez, Yaqin Zhang, and Juan C. Miñano
}

\begin{abstract}
It was previously demonstrated in [Opt. Lett. 38, 229 (2013)] that the problem of freeform surface illumination design can be converted into a nonlinear boundary problem for the elliptic Monge-Ampére equation based on the ideal source assumption. But how the Monge-Ampére equation method is affected by the characteristics of the light source and target was not discussed there. This Letter systematically analyzes the influence of discontinuity, nonconvexity, and connectivity of light source and target on the Monge-Ampére equation method and presents some intrinsic features of this design method. These features are applied in practical examples in freeform optics design. () 2014 Optical Society of America
\end{abstract}

Freeform surface illumination employs one or multiple freeform surfaces to redirect the light coming from a given light source to produce a target. There are several kinds of methods that can be used to solve this problem [1-4]. Among these design methods, the Monge-Ampére (MA) equation method may be most advanced [3,4]; however, this kind of method has been covered by a veil of mystery for a long time due to the lack of details published about it [3]. It was demonstrated in a previous publication that this design problem, which is similar to the problem of optimal mass transport, can be converted into a nonlinear boundary problem for the elliptic MA equation [5]. Establishing this kind of mathematical model for freeform surface illumination removed the veil of mystery from the MA method for the first time. In the Cartesian coordinate system, the mathematical model of collimated beam shaping is given by [ $[\underline{6}]$

$$
\left\{\begin{array}{l}
E\left(t_{x}(x, y), t_{y}(x, y)\right)|J(\mathbf{T})|=I(x, y) \\
\mathrm{BC}:\left\{\begin{array}{l}
t_{x}=t_{x}\left(x, y, z, z_{x}, z_{y}\right) \\
t_{y}=t_{y}\left(x, y, z, z_{x}, z_{y}\right)
\end{array}: \partial S_{1} \rightarrow \partial S_{2},\right.
\end{array}\right.
$$

where $I(x, y)$ is the intensity distribution of the light source, $E\left(t_{x}, t_{y}\right)$ is the irradiance distribution of the target, $S_{1}$ and $S_{2}$ denote the cross section of the source and the target illumination area, respectively, and $\partial S_{1}$ and $\partial S_{2}$ are, respectively, the boundaries of $S_{1}$ and $S_{2}$, as shown in Fig. 1. Since the MA equation is a partial differential equation of the second order, the freeform surface governed by this equation is at least geometrically smooth. The boundary condition (BC) specifies that the incident rays on $\partial S_{1}$ should be refracted by the freeform surface to $\partial S_{2}$. The first expression in the design model, which represents the conservation and redistribution of power during the beam-shaping process, is an elliptic MA equation, and this MA equation is strongly determined by $E\left(t_{x}, t_{y}\right)$ and $I(x, y)$. We have demonstrated the elegance of the MA method in freeform optics design [5-7], but we still do not know how this MA method is affected by the characteristics of the light source and target. Is it possible to achieve any prescribed beam shaping by using the MA method? If not, what are the requirements for characteristics of the light source and target? To answer these questions, we will systematically analyze the influence of discontinuity, nonconvexity, and connectivity of the light source and target on the MA method, and present some intrinsic features of the MA method in this Letter.

For brevity, let us write $E$ instead of $E\left(t_{x}, t_{y}\right)$ and $I$ instead of $I(x, y)$. Then, if we expand and simplify the first expression in Eq. (1), we can obtain

$$
E \Phi\left(z_{x x}, z_{y y}, z_{x y}, z_{x}, z_{y}, z,\right)-I=0
$$

where $\Phi=A_{1}\left(z_{x x} z_{y y}-z_{x y}^{2}\right)+A_{2} z_{x x}+A_{3} z_{y y}+A_{4} z_{x y}+$ $A_{5}$ and the coefficients $A_{i}(i=1, \ldots, 5)$ are functions of $x, y, z, z_{x}$, and $z_{y}$. We define $F=E \Phi-I$ and use the nine-point finite difference for derivatives [ 6$]$ ]. Then we can obtain a set of nonlinear equations by which the inner grid points are governed:

$$
\begin{aligned}
& E_{i, j} f\left(z_{i-1, j-1}, z_{i-1, j}, z_{i-1, j+1}, z_{i, j-1}, z_{i, j}, z_{i, j+1}, z_{i+1, j-1},\right. \\
& \left.\quad z_{i+1, j}, z_{i+1, j+1}\right)-I_{i, j}=0 .
\end{aligned}
$$

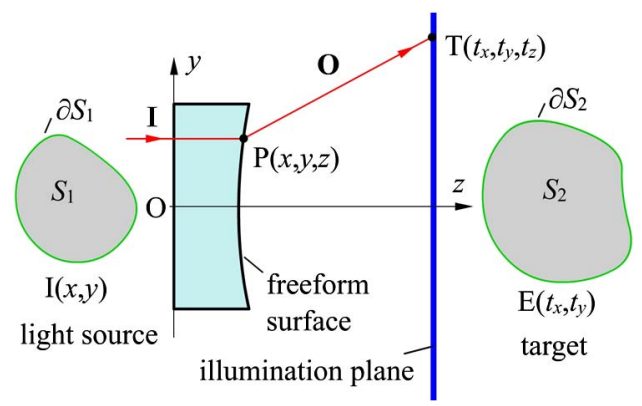

Fig. 1. Illustration of the collimated beam shaping. 
If we linearize this nonlinear problem, we get an iterative scheme for all the inner grid points, which is given by

$$
F\left(\mathbf{X}_{k}\right)+F^{\prime}\left(\mathbf{X}_{k}\right)\left(\mathbf{X}_{k+1}-\mathbf{X}_{k}\right)=0,
$$

where $\mathbf{X}_{k}$ denotes the solution obtained from the $k$ th iteration and $F^{\prime}\left(\mathbf{X}_{k}\right)$ is the Fréchet derivative of $F$ at $\mathbf{X}_{k}$. For a inner point $(i, j)$, we have a specific form of Eq. (4):

$$
\begin{aligned}
& E_{i, j}\left(0, \ldots f_{i-1, j-1}^{\prime}, 0, \ldots f_{i-1, j}^{\prime}, 0, \ldots f_{i-1, j+1}^{\prime}, 0, \ldots f_{i, j-1}^{\prime}, 0, \ldots f_{i, j}^{\prime}\right. \\
& \left.0, \ldots f_{i, j+1}^{\prime}, 0, \ldots f_{i+1, j-1}^{\prime}, 0, \ldots f_{i+1, j}^{\prime}, 0, \ldots f_{i+1, j+1}^{\prime}, 0, \ldots\right) \\
& \quad \times\left(\mathbf{X}_{k+1}-\mathbf{X}_{k}\right)=-F_{i, j}\left(\mathbf{X}_{k}\right),
\end{aligned}
$$

where $f_{i, j}^{\prime}$ denotes $\partial f / \partial z_{i, j}$. Similarly, we can tackle the boundary points and establish an iterative scheme for the mathematical model, which is written as

$$
\mathbf{A} \Delta \mathbf{X}=\mathbf{B},
$$

where $\Delta \mathbf{X}=\mathbf{X}_{k+1}-\mathbf{X}_{k}$, $\mathbf{A}$ is a square sparse matrix of dimensions $(m+1) \times(n+1), \mathbf{B}$ is an $(m+1) \times(n+1)$ dimensional column vector, and the number of variables equals $(m+1) \times(n+1)$. An initial design should be given, which can converge to a stable solution after several iterations. Here we employ a design shown in Fig. 2 as the initial value for the iterative scheme during the following analyses.

According to the numerical technique presented above, first the domain $S_{1}$ should be discretized, and then an intensity matrix has to be determined for all the inner grid points, as indicated in Eq. (3). For a given $I(x, y)$, which is an analytic function, the intensity matrix is defined by the function of each inner point. When $I(x, y)$ cannot be expressed in an analytic form (for example, $I(x, y)$ is a discontinuous function or even a bitmap recorded by a camera), the intensity matrix can be obtained by data interpolation. Figure 3 gives the results of these two cases. Here we assume the target is a uniform elliptical pattern. In Fig. 3(a), the incident beam has a Gaussian distribution, and $I(x, y)$ is a discontinuous function in Fig. 3(b). The targets are successfully achieved, and smooth surfaces are obtained. The results show clearly that the discontinuity of $I(x, y)$ has no influence on the MA method.

The domain $S_{1}$ is a square, which, of course, is a convex set. The perfect results shown in Fig. 3 indicate the MA method can work for a convex set. If $S_{1}$ is not a convex set, then what will happen in the design? Figure 4(a) gives a domain $S_{11}$, which is not a convex set. Since $\overline{S_{11}}$ is

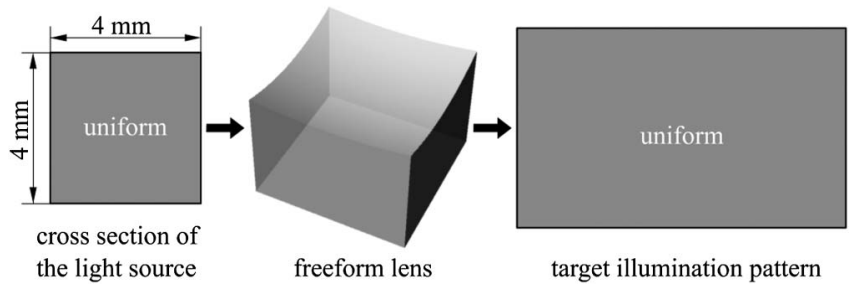

Fig. 2. In the initial design, the incident beam with a uniform intensity distribution can be reshaped by the freeform lens to produce a uniform rectangular pattern.
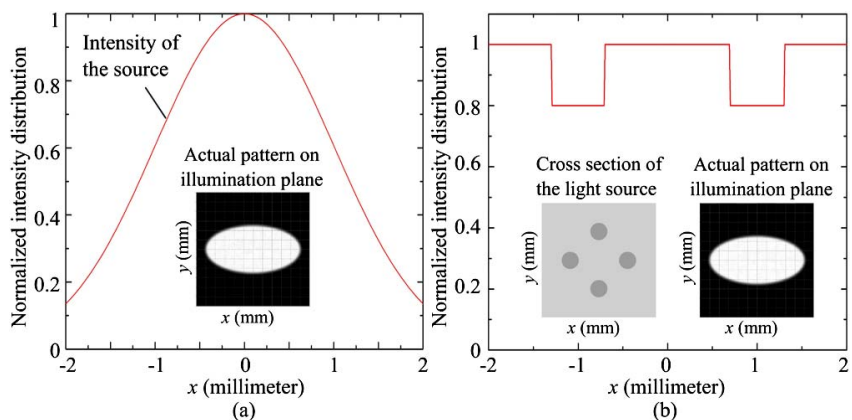

Fig. 3. Intensity distribution of the light source along the line $y=0 \mathrm{~mm}$, and the obtained illumination pattern. (a) $I(x, y)$ is an analytic function and (b) $I(x, y)$ is a discontinuous function.

not a rectangle or a circle, it is actually very hard to execute the discretization operation for such an irregular domain. To solve this problem, we create a regular domain $S_{1}$, which is an addition of domain $S_{11}$ with domain $S_{12}$, as shown in Fig. 4(a). And we let the intensity of $S_{12}$ equal zero. Then we can execute the discretization operation for $S_{1}$, which is a rectangle. According to the $\mathrm{BC}$, the incident rays on $\partial S_{11}$ should be refracted by the freeform surface to $\partial S_{2}$. With the fill operation, will the incident rays on the curve $l_{1}$ still be refracted to $\partial S_{2}$ ? In Eq. (1), the quantity $|J(\mathbf{T})|$ represents the expansion (or contraction) of an infinitesimal tube of rays due to the refraction of the freeform surface. Namely, the infinitesimal tube of rays will converge to a point, a straight line, or a curve when $|J(\mathbf{T})|=0$. Since the intensity of $S_{12}$ equals zero and $E\left(t_{x}, t_{y}\right) \neq 0,|J(\mathbf{T})|$ will approach zero during the iteration. Consequently, both the rays on $l_{1}$ and the rays within $S_{12}$ are refracted to the boundary of the illumination pattern, as shown in Fig. 5. A smooth freeform surface is obtained, and the target illumination is achieved. Figures 4 and 5 show clearly that the MA method can also work for a nonconvex set. According to the results of these two cases, we find that nonconvexity of the domain $S_{1}$ of the light source has no influence on the MA method, and the iterative scheme can still converge quickly and successfully. Also, the fill operation provides us an effective tool to tackle the designs where $S_{1}$ is not a regular domain.

A simply connected domain is a path-connected domain where one can continuously shrink any simple closed curve into a point while remaining in the domain. Obviously, the domain $S_{1}$ shown in Fig. 3(b) is simply connected, and the results obtained by the MA method are perfect. If the domain $S_{1}$ is multiply connected [for example, there is a hole in the domain shown in Fig. 4(b)], then is it possible to employ a smooth freeform surface to produce the uniform elliptical pattern?

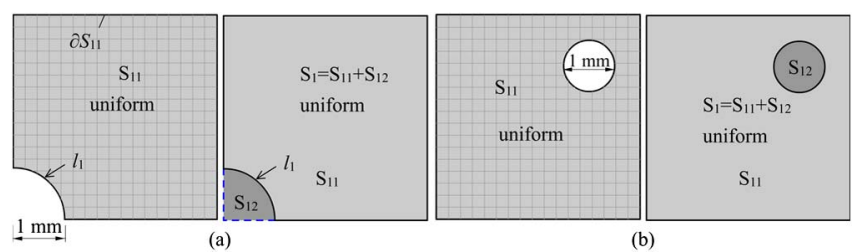

Fig. 4. (a) $S_{11}$ is a nonconvex set, and $S_{1}$ is a regular domain after fill operation and (b) $S_{11}$ is mutiply connected, and $S_{1}$ is simply connected after fill operation. 

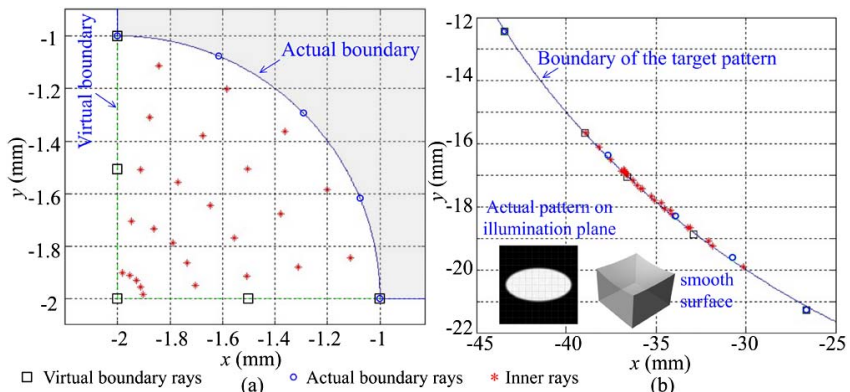

Fig. 5. (a) Incident rays of the source and (b) the intercept points of the incident rays on the target plane.

Similarly, we create a simply connected domain $S_{1}$, which is an addition of domain $S_{11}$ with domain $S_{12}$, and assume that the intensity of $S_{12}$ equals zero, as shown in Fig. 4(b). Figure 6 gives the results of this design achieved by the MA method. Since the intensity of $S_{12}$ equals zero, all the rays within $S_{12}$ almost converge to a curve, as shown in Fig. 6(b). The smooth surface is obtained, and the elliptical pattern is achieved. The results show clearly that the connectivity of $S_{1}$ has no influence on the MA method. We have discussed the influence of characteristics of the light source on the MA method. Next, we will explore how this method is affected by characteristics of the target.

According to the discretization shown in Eq. (3), an irradiance matrix should be similarly determined for all the inner grid points to solve those nonlinear equations. For a given $E\left(t_{x}, t_{y}\right)$ which is an analytic function, the irradiance matrix is calculated by the function of the intercept point of each inner ray on the target plane. When $E\left(t_{x}, t_{y}\right)$ is a discontinuous function [for example, a target contains the letters "UPM" on an elliptical background with the irradiance ratio of 2 (the letters) to 1 (background) to zero (outside)], the irradiance matrix can be determined by data interpolation with the intercept points. Figure 3 shows the result for a uniform elliptical pattern, and Fig. 7(a) gives the result of the "UPM" logo lens. The target $\left.\overline{E\left(t_{x}\right.}, t_{y}\right)$ of the design shown in Fig. $7(\mathrm{a})$ is a discontinuous function. Obviously, whether $\left.\overline{E\left(t_{x}\right.}, t_{y}\right)$ is an analytic function or not, the MA method is not affected by the discontinuity of $E\left(t_{x}, t_{y}\right)$ and can still work very well.

The boundary of the pattern shown in Fig. 7(a) is an ellipse, and the domain defined by this boundary, of course, is a convex set. Figure 7(b) gives the result of a design where the domain $S_{2}$ is a nonconvex set and the incident beam in the design has a Gaussian distribution. It shows

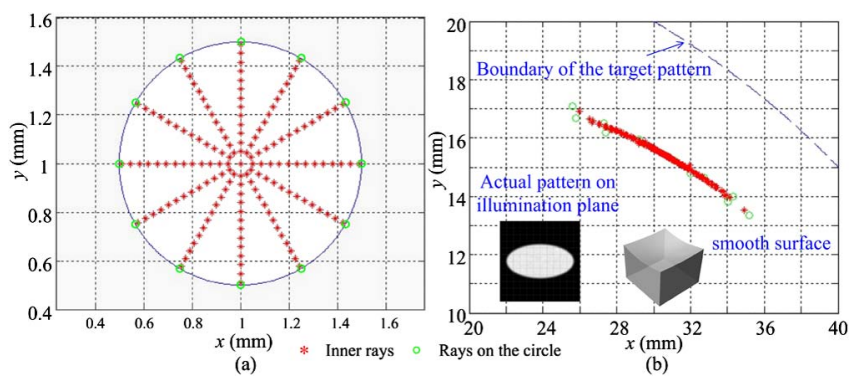

Fig. 6. (a) Incident rays of the source and (b) the intercept points of the incident rays on the target plane.

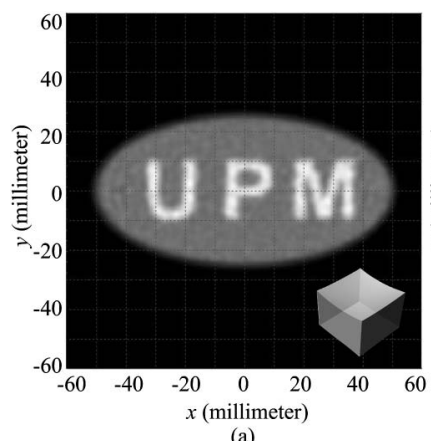

(a)

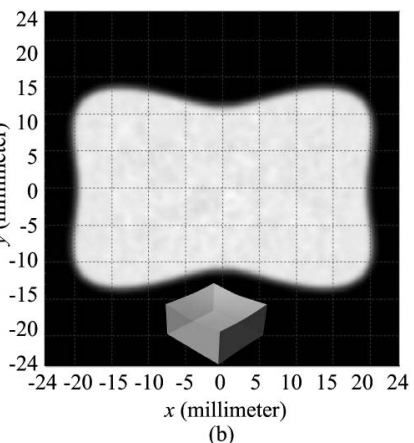

(b)
Fig. 7. (a) $E\left(t_{x}, t_{y}\right)$ is a discontinuous function and (b) $S_{2}$ is a nonconvex set.

clearly that the MA method also works very well and the target is achieved. The concavity and convexity of the domain $S_{2}$ of the target illumination has no influence on the MA method. The $\mathrm{BC}$ requires the boundary rays on $\partial S_{1}$ to be refracted by the surface to $\partial S_{2}$. Namely, the intercept points of the boundary rays on the target plane should satisfy the expression of $\partial S_{2}$. Thus, generally we need to obtain an expression of $\partial S_{2}$ before imposing the $\mathrm{BC}$ on the MA equation.

As presented above, we can still obtain perfect results when the domain $S_{1}$ of the light source is multiply connected. What will happen in the design if the domain $S_{2}$ of the target is multiply connected? Can we still use a smooth surface to produce the target? Figure $8(\mathrm{~b})$ gives a target pattern that is multiply connected. Here, the irradiance of $S_{21}$, of course, equals zero. The domain $S_{1}$ of the light source is simply connected, and the intensity distribution is uniform, as shown in Fig. 8(a). If we use the initial design given in Fig. 2 for the iteration scheme, we can calculate the intercept points of all the incident rays on the illumination plane by tracing rays with the initial design. Then the irradiance matrix can be determined by the intercept points of the incident rays. If we assume the incident rays contained in $S_{11}$ are refracted to $S_{21}$, we observe that $E_{i, j}$ corresponding to these incident rays equals zero. In this case, the coefficients contained in Eq. (4) will equal zero, and obviously the linear problem given in Eq. (6) is unsolvable. That is, we cannot obtain a smooth surface governed by the MA equation for such a target. Therefore, it is not possible to use the MA method to design a smooth surface when the domain $S_{2}$ of the target illumination is multiply connected.

Although it is impossible to produce the target pattern shown in Fig. 8(b) by using a smooth surface, this target can still be achieved by the MA method. We can divide $S_{2}$ into two separate nonconvex domains $S_{22}$ and $S_{23}$, which are not multiply connected anymore, as shown

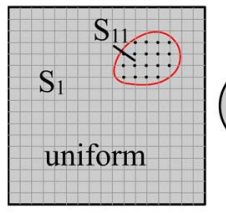

(a)

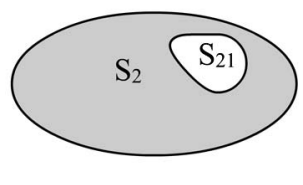

(b)

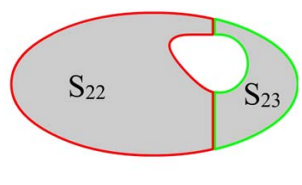

(c)
Fig. 8. (a) Cross section of the light source, (b) a multiply connected domain $S_{2}$, and (c) two separate nonconvex domains $S_{22}$ and $S_{23}$. 
(a)

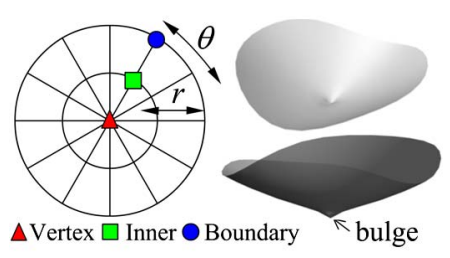

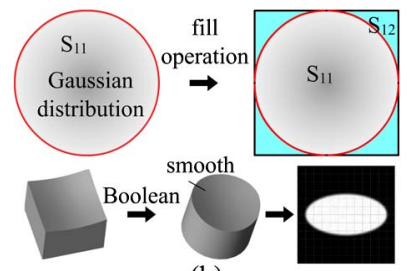

(b)
Fig. 9. (a) Discretization operation and bulge at the vertex and (b) a smooth surface with a circular aperture can be obtained by the fill operation.

in Fig. 8(c). Then these two target patterns can be separately achieved by the MA method, and two separate smooth surfaces can be obtained. However, the freeform surface composed of these two separate smooth surfaces is not geometrically smooth. Determining how to achieve such a design is beyond the scope of this Letter.

Next, two application examples will be given to illustrate the practical interest of the new features of the MA method. In most applications of collimated beam shaping, the incident beam usually has a circular cross section. In addition, the circular aperture of a freeform optics preferred, because the circular aperture will make freeform optics much easier to fabricate. To facilitate design and fabrication, we would thus like to establish a mathematical model of collimated beam shaping in the cylindrical coordinate system, which is similar to the one given in Eq. (1). However, as it is different from the discretization operation in the Cartesian coordinate system, we will obtain three kinds of grid points: the inner grid point, the boundary point, and the vertex of the freeform surface in the cylindrical coordinate system, as shown in Fig. 9(a). The inner grid point should satisfy the MA equation, and the boundary point should satisfy the BC. However, the vertex does not satisfy either the MA equation or the BC. Although the vertex is carefully treated, sometimes the iteration still fails to converge due to an unexpected bulge at the vertex of the freeform surface, shown in Fig. 9(a). Fortunately, we can use the design model given in Eq. (1) together with the fill operation and Boolean operation to solve this problem, as shown in Fig. 9(b). The target is successfully achieved, and the circular aperture is also obtained. The fill operation can avoid complications associated with singularities in the cylindrical coordinate system.

Due to the excellent characteristics that can be used to achieve a large angle of deflection, high energy efficiency, and a compact design, the total internal reflection (TIR) lens is very popular in general lighting $[\underline{1}, \underline{8}, \underline{9}]$. Currently, the TIR lens is mostly used to produce rotational illumination, and it may still be a challenge to design a TIR lens for nonrotational illumination. However, the excellent features of the MA method disclosed above give us a good chance of easily solving this problem. The second example is a TIR freeform lens, which can produce a nonrotational illumination, as shown in Fig. 10 . Figure 10(b) gives the irradiance distribution produced by the TIR collimator, whose back surface is designed to perfectly collimate the rays, and the nonrotational pattern is shown in Fig. 10(c).

This Letter demonstrates the influence of the characteristics of a light source and target on the

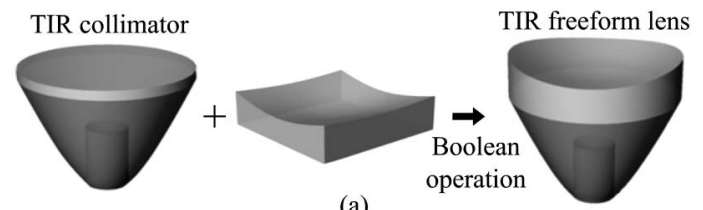

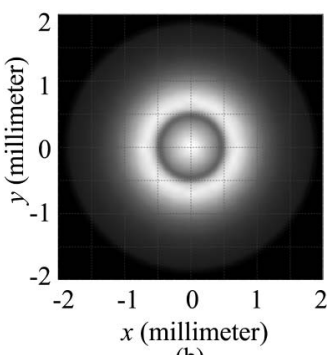

(b) (a)

(c)

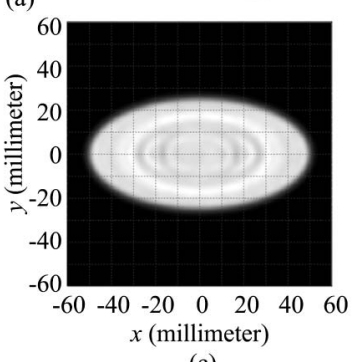

Fig. 10. Rays coming from a Lambertian point source are redirected by the TIR freeform lens to produce a uniform elliptical pattern. (a) The procedure of designing the TIR freeform lens, (b) the pattern produced by the TIR collimator in the near field, and (c) the pattern produced by the TIR freeform lens on the illumination plane.

Monge-Ampére equation method in freeform optics design and presents some intrinsic features of the MA method. The design examples perfectly show practical application of these features of the MA method in freeform optics design. This work is important from both mathematical and practical standpoints, and the new features of the MA method presented in this Letter are also correct in the case of a point source, as demonstrated in [5].

This work was carried out during the tenure of an ERCIM "Alain Bensoussan" Fellowship Programme. The research leading to the results has received funding from the European Union Seventh Framework Programme (FP7/2007-2013) under Grant Agreement No. 246016. Authors thank the European Commission (SMETHODS: FP7-ICT-2009-7 Grant Agreement No. 288526, NGCPV: FP7-ENERGY.2011.1.1 Grant Agreement No. 283798), the Spanish Ministries (ENGINEERING METAMATERIALS: CSD2008-00066, SEM: TSI-020302-2010-65, SUPERRESOLUCION: TEC201124019, SIGMAMODULOS: IPT-2011-1441-920000, PMEL: IPT-2011-1212-920000), and UPM (Q090935C59) for the support given to the research activity of the UPM-Optics Engineering Group, making the present work possible.

1. J. Bortz, N. Shatz, and M. Keuper, Proc. SPIE 5529, 8 (2004).

2. F. R. Fournier, W. J. Cassarly, and J. P. Rolland, Opt. Express 18, 5295 (2010).

3. H. Ries and J. Muschaweck, J. Opt. Soc. Am. A 19, 590 (2002).

4. V. Oliker, J. Opt. Soc. Am. A 24, 3741 (2007).

5. R. M. Wu, L. Xu, P. Liu, Y. Q. Zhang, Z. R. Zheng, H. F. Li, and X. Liu, Opt. Lett. 38, 229 (2013).

6. R. M. Wu, P. Liu, Y. Q. Zhang, Z. R. Zheng, H. F. Li, and X. Liu, Opt. Express 21, 20974 (2013).

7. R. M. Wu, K. Li, P. Liu, Z. R. Zheng, H. F. Li, and X. Liu, Appl. Opt. 52, 5272 (2013).

8. Z. R. Zheng, X. Hao, and X. Liu, Appl. Opt. 48, 6627 (2009).

9. J. J. Chen, T. Y. Wang, K. L. Huang, T. S. Liu, M. D. Tsai, and C. T. Lin, Opt. Express 20, 10984 (2012). 